\title{
Global solutions to the volume-preserving mean-curvature flow
}

\author{
Luca Mugnai $^{1}$ - Christian Seis ${ }^{2} \cdot$ Emanuele Spadaro $^{1}$
}

Received: 24 February 2015 / Accepted: 27 October 2015 / Published online: 2 February 2016

(C) The Author(s) 2016. This article is published with open access at Springerlink.com

\begin{abstract}
In this paper, we construct global distributional solutions to the volume-preserving mean-curvature flow using a variant of the time-discrete gradient flow approach proposed independently by Almgren et al. (SIAM J Control Optim 31(2):387-438, 1993) and Luckhaus and Sturzenhecker (Calc Var Partial Differ Equ 3(2):253-271, 1995).
\end{abstract}

Keywords Mean-curvature flow · Volume preserving · Volume constraint · Global solutions - Time discretization

Mathematics Subject Classification $\quad 53 \mathrm{C} 44 \cdot 58 \mathrm{E} 12 \cdot 65 \mathrm{M} 06$

\section{Introduction}

A family of open sets with smooth boundary $\left\{E_{t}\right\}_{0 \leq t \leq T}$ in $\mathbb{R}^{n}$ is said to move according to volume-preserving mean-curvature flow if the motion law, expressed as an evolution equation for the boundaries $\partial E_{t}$, takes the form

$$
v=\langle H\rangle-H \text { on } \partial E_{t},
$$

Communicated by L. Ambrosio.

Emanuele Spadaro

spadaro@mis.mpg.de

Luca Mugnai

mugnai@mis.mpg.de

Christian Seis

seis@iam.uni-bonn.de

1 Max-Planck-Institut für Mathematik in den Naturwissenschaften, Inselstraße 22, 04103 Leipzig, Germany

2 Institut für Angewandte Mathematik Universität Bonn, Endenicher Allee 60, 53115 Bonn, Germany 
for all $t \in[0, T]$. Here, at any point $x$ on $\partial E_{t}, v(x)$ denotes the velocity component normal to the boundary, in the direction of the outer normal, $H(x)$ is the scalar mean curvature (with the sign convention that $H$ is positive for balls, see the next section), and the brackets $\langle\cdot\rangle$ denote the average of a quantity over the boundary of $E_{t}$.

It is immediately verified that the volume of the sets $E_{t}$ (i.e., its $n$-dimensional Lebesgue measure, denoted by $\left.\left|E_{t}\right|\right)$ is indeed preserved under the smooth flow (1.1) because

$$
\frac{d}{d t}\left|E_{t}\right|=\int_{\partial E_{t}} v d \mathcal{H}^{n-1} \stackrel{(1.1)}{=} 0 .
$$

And thus, upon rescaling variables, we may assume that $\left|E_{t}\right|=\left|E_{0}\right|=1$ for any $t \in[0, T]$. Moreover, the perimeter of the sets $E_{t}$ is decreasing because

$$
\frac{d}{d t} \mathcal{H}^{n-1}\left(\partial E_{t}\right)=\int_{\partial E_{t}} v H d \mathcal{H}^{n-1} \stackrel{(1.1)}{=}-\int_{\partial E_{t}} v^{2} d \mathcal{H}^{n-1} \leq 0 .
$$

During a typical evolution, a volume-preserving mean-curvature flow exhibits singularities of different kinds, even in the case of smooth initial data. These singularities correspond to changes in the topology of the configuration and include shrinkage of islands to points and disappearance, collisions and merging of neighboring islands, pinch-offs etc... If the topology changes, the boundary of the evolving set looses regularity and, as a consequence, the formulation (1.1) of the evolution law is inadequate. The goal of the present work is the construction of a notion of a weak solution to the volume-preserving mean-curvature flow that is global in time and thus overcomes these singular moments.

Several solutions to volume-preserving mean-curvature flow have been proposed in the literature: existence and uniqueness of a global in time smooth solution and its convergence to a sphere is shown in [17,22] for smooth convex initial data and in $[15,24]$ for initial data close to a sphere (for further related results see $[4,7,8]$ and the references therein). In principle, these results also yield local in time existence and uniqueness of smooth solutions. In [30] and [31], the authors consider level-set and diffusion-generated solutions for the purpose of numerical studies. In Bellettini et al. [5], construct solutions for the volume-preserving anisotropic mean-curvature [or anisotropic variant of (1.1)] for convex sets using a method similar to ours (that will be outlined below). In this paper, it is also shown that the solution (the so-called flat flow) is unique and coincides with regular solutions when the latter are defined. We also mention a mean field approximation approach to the volume preserving mean-curvature flow as developed in $[1,6,12]$.

It is well-known that volume-preserving mean-curvature can be (formally) interpreted as the $L^{2}$-gradient flow of the perimeter functional for configurations with a fixed volume, see, e.g., [29, Sect. 2]. This gradient flow structure, however, is for the purpose of well-posedness results impracticable, since the $L^{2}$ (geodesic) distance is degenerate in the sense that two well separated configurations may have zero $L^{2}$ distance [28]. In the present manuscript, we follow the method proposed independently by Almgren et al. [2] and Luckhaus and Sturzenhecker [26] in the study of (forced) mean-curvature flows to bypass this difficulty. The authors consider an implicit time-discretization of the flow, which comes as a gradient flow of the perimeter functional with respect to a new non-degenerate distance function that approximates the $L^{2}$ distance. The limiting time-continuous flow constructed with this method is usually referred to as the flat flow. The main difference between the present work and $[2,26]$ relies on the non-locality of the volume-preserving mean-curvature flow. As an immediate consequence, there is no maximum-principle available for (1.1). Also related to this aspect, there is the problem of proving the consistency of the scheme, i.e. the coincidence of the flat volume-preserving mean-curvature flow with the smooth one when the latter exists. 
Under some assumption on the Lagrange multiplier of the flow, the consistency can be inferred from the arguments in [2], but we do not know if these conditions are generally satisfied and we do not discuss further the problem of the consistency in this paper. A more detailed discussion on the different features of the flows in $[2,26]$ and the one considered in the present manuscript will follow in Sect. 3 below.

We conclude this subsection with a short discussion on the background of this evolution. Volume-preserving mean-curvature flow can be considered as a simplified model for attachment-limited kinetics, and as such it plays an important role in the study of solidification processes, where solid islands grow in an undercooled liquid of the same substance. In such situations, solid particles melt at high-curvature regions and simultaneously precipitate at low-curvature regions, while the total mass of the solid remains essentially constant $[11,32,33]$. In this way, the total surface area of solid islands is decreasing, and thus, this process leads to the growth of larger islands at the expense of smaller ones: a phenomenon called coarsening [29]. In general, solidification processes are mathematically often modelled by Mullins-Sekerka equations (or a Stefan problem), where the Gibbs-Thompson relation is modified by a kinetic drift term [21,25], and their phase-field counterparts respectively [9]. This model allows for both attachment kinetics (kinetic drift) and bulk diffusion (MullinsSekerka/Stefan). It turns out that attachment kinetics is the relevant mass transport mechanism in earlier stages of the evolution while bulk diffusion predominates the later stages [13]. In a certain sense, volume-preserving mean-curvature flow naturally arises as the singular limit of this more general solidification model in the regime of vanishing bulk diffusion. More recently, variants of volume-preserving mean curvature flow were also applied in the context of shape recovery in image processing [10].

The article is organized as follows: in Sect. 2 we fix the notation and state the main results of the paper, which are then proved in Sects. 3 and 4 and are the existence of flat volumepreserving mean-curvature flows and the existence of distributional solutions, respectively.

\section{Statements of the main results}

\subsection{Notation}

For any Lebesgue measurable set $E \subset \mathbb{R}^{n}$, we denote by $|E|$ the $n$-dimensional Lebesgue measure of $E$ and by $\chi_{E}$ the characteristic function of $E$, i.e. $\chi_{E}(x)=0$ if $x \notin E$ and $\chi_{E}(x)=1$ if $x \in E$. The perimeter of $E$ in an open set $\Omega \subset \mathbb{R}^{n}$ is defined as

$$
\operatorname{Per}(E, \Omega):=\sup \left\{\int_{E} \operatorname{div} \varphi d x: \varphi \in C_{c}^{1}\left(\Omega ; \mathbb{R}^{n}\right) \text { with } \sup _{\Omega}|\varphi| \leq 1\right\},
$$

and we write $\operatorname{Per}(E):=\operatorname{Per}\left(E, \mathbb{R}^{n}\right)$. If the latter quantity is finite, we will call $E$ a set of finite perimeter. In the case that $E$ is an open set with $\partial E$ of class $C^{1}$, we simply have $\operatorname{Per}(E, \Omega)=\mathcal{H}^{n-1}(\partial E \cap \Omega)$ and $\operatorname{Per}(E)=\mathcal{H}^{n-1}(\partial E)$. The reduced boundary of a set of finite perimeter $E$ is denoted by $\partial^{*} E$, cp. [16, Sect. 5.7], and for the unit outer normal to $E$ we write $v_{E}$. The tangential divergence of a vector field $\Psi \in C^{1}\left(\mathbb{R}^{n} ; \mathbb{R}^{n}\right)$ with respect to $\partial E$ is defined by $\operatorname{div}_{\partial E} \Psi:=\operatorname{div} \Psi-v_{E} \cdot \nabla \Psi v_{E}$. We say that a set of finite perimeter $E$ has a (generalized) mean-curvature $H_{E} \in L^{1}\left(\partial^{*} E, d \mathcal{H}^{n-1}\right)$ provided that

$$
\int_{\partial^{*} E} \operatorname{div}_{\partial E} \Psi d \mathcal{H}^{n-1}=\int_{\partial^{*} E} \Psi \cdot v_{E} H_{E} d \mathcal{H}^{n-1} \text { for all } \Psi \in C_{c}^{1}\left(\mathbb{R}^{n} ; \mathbb{R}^{n}\right) .
$$

Observe that with this sign convention it is $H_{B_{R}} \equiv \frac{n-1}{R}$. 
We write $\operatorname{sd}_{F}$ for the signed distance from a Lebesgue measurable set $F$, with the convention that $\operatorname{sd}_{F}$ is negative inside $F$, i.e.,

$$
\operatorname{sd}_{F}(x)= \begin{cases}\operatorname{dist}(x, F) & \text { for } x \in F^{c}, \\ -\operatorname{dist}\left(x, F^{c}\right) & \text { for } x \in F,\end{cases}
$$

Here, $F^{c}:=\mathbb{R}^{n} \backslash F$ denotes the complement set of $F$, and the distance from a set $F$ is by definition

$$
\operatorname{dist}(x, F)=\inf _{y \in F}|x-y| .
$$

We will sometimes use the notation $\mathrm{d}_{F}=\left|\mathrm{sd}_{F}\right|$.

By $[t]$ we denote the integer part of a real number $t$, that is the biggest integer $m$ such that $m \leq t$.

Finally, we denote by $c_{n}, C_{n}$ positive constants that depend on the space dimension only. Moreover, $c_{n, 0}, C_{n, 0}$, and $C_{n, 0, T}$ are constants that may additionally depend on the initial data or the time $T>0$. During the computations, the value of these constants may change from line to line. However, for the sake of clarity we need to keep track of the dimensional constant in Proposition 3.2, therefore we make an exception to the above convention and denote it by $\gamma_{n}$. The volume of the $n$-dimensional unit ball will be denoted by $\omega_{n}$, and thus its surface area is $n \omega_{n}$.

\subsection{Approximate solutions}

In this paper we introduce a notion of global flat solution to the volume-preserving meancurvature flow which is based on the implicit time discretization of (1.1) in the spirit of Almgren et al. [2] and Luckhaus and Sturzenhecker [26]. That is, we consider a time-discrete gradient flow for the perimeter functional. For this purpose, we define

$$
\mathcal{F}_{h}(E, F):=\operatorname{Per}(E)+\frac{1}{h} \int_{E} \operatorname{sd}_{F} d x+\frac{1}{\sqrt{h}}|| E|-1|,
$$

for any two sets of finite perimeter $E$ and $F$ in $\mathbb{R}^{n}$. Here, $h$ is a positive small number that plays the role of the time step of approximate solutions. The second term in the above functional approximates the degenerate $L^{2}$ geodesic distance on the configuration space of hypersurfaces. The functional differs from the one considered in the original papers $[2,26]$ only in the last term: a weak penalization that favors unit-volume of minimizing sets.

Definition 2.1 Let $E_{0}$ be a set of finite perimeter with $\left|E_{0}\right|=1$, and $h>0$. Let $\left\{E_{k h}^{(h)}\right\}_{k \in \mathbb{N}}$ be a sequence of sets defined iteratively by

$$
E_{0}^{(h)}=E_{0} \quad \text { and } \quad E_{k h}^{(h)} \in \arg \min _{E \subset \mathbb{R}^{n}}\left\{\mathcal{F}_{h}\left(E, E_{(k-1) h}^{(h)}\right)\right\} \quad \text { for } k \geq 1 .
$$

We furthermore define

$$
E_{t}^{(h)}:=E_{k h}^{(h)} \text { for any } t \in[k h,(k+1) h),
$$

and call $\left\{E_{t}^{(h)}\right\}_{t \geq 0}$ an approximate flat solution to the volume-preserving mean-curvature flow with initial datum $E_{0}$.

The existence of minimizers $E_{k h}^{(h)}$ and thus the existence of an approximate flat solution is guaranteed by Lemma 3.1 below. Incorporating the volume constraint in a soft way into the energy functional rather than imposing a hard constraint on the admissible sets has the 
advantage that we are free to chose arbitrary competitors, most notably in the derivation of density estimates. Thanks to the penalizing factor $1 / \sqrt{h}$, the constraint becomes active in the limit $h \downarrow 0$. Even more can be shown: the number of time steps in which approximate solutions violate the volume constraint $\left|E_{t}^{(h)}\right|=1$ can be bounded uniformly in $h$, cf. Corollary 3.10 below. A similar functional including a soft volume constraint was recently considered by Goldman and Novaga [20] in the study of a prescribed curvature problem.

\subsection{Main results}

We can now state our main results. The first one is a convergence result for approximate solutions.

Theorem 2.2 (Existence of flat flows) Let $E_{0}$ be a bounded set of finite perimeter with $\left|E_{0}\right|=1$ and, for any $h>0$, let $\left\{E_{t}^{(h)}\right\}_{t \geq 0}$ be an approximate flat solution to the volumepreserving mean-curvature flow with initial datum $E_{0}$. Then, there exists a family of sets of finite perimeter $\left\{E_{t}\right\}_{t \geq 0}$ and a subsequence $h_{k} \downarrow 0$ such that

$$
\left|E_{t}^{\left(h_{k}\right)} \Delta E_{t}\right| \rightarrow 0 \text { for a.e. } t \in[0,+\infty),
$$

and, for every $0 \leq s \leq t$,

$$
\begin{gathered}
\left|E_{t}\right|=1, \\
\left|E_{t} \Delta E_{s}\right| \leq C_{n, 0}|t-s|^{1 / 2}, \\
\operatorname{Per}\left(E_{t}\right) \leq \operatorname{Per}\left(E_{s}\right) .
\end{gathered}
$$

Our next statement is the existence of a distributional solution in the sense of Luckhaus and Sturzenhecker [26] to the volume-preserving mean-curvature flow under the hypothesis that the perimeters of the approximate solutions converge to the perimeter of the limiting solutions identified in the previous theorem.

Theorem 2.3 (Existence of distributional solutions) Suppose that $n \leq$ 7. Let $\left(h_{k}\right)_{k \in \mathbb{N}}$ and $\left\{E_{t}\right\}_{t \geq 0}$ be as in Theorem 2.2. For any $T>0$, if

$$
\lim _{k \rightarrow \infty} \int_{0}^{T} \operatorname{Per}\left(E_{t}^{\left(h_{k}\right)}\right) d t=\int_{0}^{T} \operatorname{Per}\left(E_{t}\right) d t,
$$

then $\left\{E_{t}\right\}_{0 \leq t<T}$ is a distributional solution to the volume-preserving mean-curvature flow with initial datum $E_{0}$ in the following sense:

(1) for almost every $t \in[0, T)$ the set $E_{t}$ has (generalized) mean curvature in the sense of (2.1) satisfying

$$
\int_{0}^{T} \int_{\partial^{*} E_{t}}\left|H_{E_{t}}\right|^{2}<+\infty
$$

(2) there exists $v: \mathbb{R}^{n} \times(0, T) \rightarrow \mathbb{R}$ with $\left.v(\cdot, t)\right|_{\partial^{*} E_{t}} \in L_{0}^{2}\left(\partial^{*} E_{t}, d \mathcal{H}^{n-1}\right)$ for a.e. $t \in(0, T)$ and $\int_{0}^{T} \int_{\partial^{*} E_{t}} v^{2} d \mathcal{H}^{n-1} d t<+\infty$ such that

$$
\begin{gathered}
-\int_{0}^{T} \int_{\partial^{*} E_{t}} v \phi d \mathcal{H}^{n-1} d t=\int_{0}^{T} \int_{\partial^{*} E_{t}}\left(H_{E_{t}} \phi-\lambda \phi\right) d \mathcal{H}^{n-1} d t, \\
\int_{0}^{T} \int_{E_{t}} \partial_{t} \phi d x d t+\int_{E_{0}} \phi(0, \cdot) d x=-\int_{0}^{T} \int_{\partial^{*} E_{t}} v \phi d \mathcal{H}^{n-1} d t,
\end{gathered}
$$


for every $\phi \in C_{c}^{1}\left([0, \infty) \times \mathbb{R}^{n}\right)$, where

$$
\lambda(t):=\frac{1}{\mathcal{H}^{n-1}\left(\partial^{*} E_{t}\right)} \int_{\partial^{*} E_{t}} H_{E_{t}} d \mathcal{H}^{n-1} \text { for a.e. } t \in[0, T) .
$$

In the second part of the theorem, $L_{0}^{2}$ is the set of all $L^{2}$ functions with zero mean.

Note that (2.4) is a weak formulation of (1.1), while (2.5) establishes the link between $v$ and the velocity of the boundaries of $E_{t}$. It is straightforward to check that smooth solutions of (1.1) satisfy (2.4) and (2.5).

The restriction on the dimension $n \leq 7$ is technical and is needed in the proof of Corollary 4.2 where the Bernstein theorem for minimal surfaces is exploited.

\section{Flat volume-preserving mean-curvature flows}

In this section we prove the first main result in Theorem 2.2. We follow quite closely Luckhaus and Sturzenhecker [26], providing all the details for the readers' convenience.

\subsection{Existence of approximate solutions}

We start remarking that

$$
\int_{E} \operatorname{sd}_{F} d x=\int_{E \Delta F} \mathrm{~d}_{F} d x-\int_{F} \mathrm{~d}_{F} d x .
$$

The existence of the approximate flat solutions is guaranteed by the following lemma.

Lemma 3.1 Let $F \subset \mathbb{R}^{n}$ be a bounded set of finite perimeter. For every $h>0$, there exists a minimizer $E$ of $\mathcal{F}_{h}(\cdot, F)$ and, moreover, E satisfies the discrete dissipation inequality

$$
\operatorname{Per}(E)+\frac{1}{h} \int_{E \Delta F} \mathrm{~d}_{F} d x+\frac{1}{\sqrt{h}}|| E|-1| \leq \operatorname{Per}(F)+\frac{1}{\sqrt{h}}|| F|-1| .
$$

Proof Since $F$ is an admissible competitor, we obtain by (3.1) that

$$
0<\inf _{\tilde{E}} \mathcal{F}_{h}(\tilde{E}, F)+\frac{1}{h} \int_{F} \mathrm{~d}_{F} d x \leq \operatorname{Per}(F)+\frac{1}{\sqrt{h}}|| F|-1|<\infty .
$$

Let $\left\{E_{\nu}\right\}_{v \in \mathbb{N}}$ denote a minimizing sequence of $\mathcal{F}_{h}(\cdot, F)$. Without loss of generality we may assume that $E_{v} \subset \subset B_{R}$ for a suitable $R>0$. Since $\left\{\chi_{E_{v}}\right\}_{v \in \mathbb{N}}$ is bounded in $B V\left(B_{R}\right)$, there exists a subsequence (not relabeled) that converges weakly to a function $\chi$ in $B V\left(B_{R}\right)$, and thus strongly in $L^{1}\left(B_{R}\right)$. In particular, $\chi$ is the characteristic function of some set of finite perimeter $E$. Since $\chi_{\tilde{E}} \mapsto \int_{\tilde{E}} \operatorname{sd}_{F} d x$ is continuous and the perimeter is lower semicontinuous with respect to $L^{1}$ convergence, it follows that

$$
\mathcal{F}_{h}(E, F) \leq \liminf _{\nu \uparrow \infty} \mathcal{F}_{h}\left(E_{\nu}, F\right)=\inf _{\tilde{E} \subset \mathbb{R}^{n}} \mathcal{F}_{h}(\tilde{E}, F) .
$$

Therefore, $E$ minimizes $\mathcal{F}_{h}(\cdot, F)$ and (3.2) follows from (3.3).

By standard results on minimal surfaces (see [27]), it holds that the minimizers $E$ of $\mathcal{F}_{h}(\cdot, F)$ can be chosen to be closed subsets with $\partial E$ of class $C^{1, \alpha}$ up to a (relatively closed) singular set of dimension at most $n-7$. Using the Euler-Lagrange equation for $\mathcal{F}_{h}(\cdot, F)$, one can also show that the regular part of the boundary $\partial E$ is actually $C^{2, \kappa}$ (cp. Lemma 3.7). 


\section{2 $L^{\infty}$ and $L^{1}$-estimates}

Our next statement gives a uniform bound on the distance between the boundary of the minimizing set and the boundary of the reference set.

Proposition 3.2 ( $L^{\infty}$-estimate) There exists a dimensional constant $\gamma_{n}>0$ with the following property. Let $F \subset \mathbb{R}^{n}$ be a bounded set of finite perimeter and let $E$ be a minimizer of $\mathcal{F}_{h}(\cdot, F)$. Then,

$$
\sup _{E \Delta F} \mathrm{~d}_{F} \leq \gamma_{n} \sqrt{h}
$$

Proof The proof of this proposition is based on the density estimates for one-side minimizing set which for readers' convenience we prove in the Appendix 1. We claim indeed that the statement holds with

$$
\gamma_{n}=\max \left\{3, \frac{4 n \omega_{n}}{c_{n}}\right\},
$$

where $c_{n}$ is the dimensional constant in Lemma 4.4. The argument is by contradiction. Let $c>\max \left\{3, \frac{4 n \omega_{n}}{c_{n}}\right\}$ and let $x_{0} \in F \Delta E$ contradict (3.4) with $\gamma_{n}$ replaced by $c$. Without loss of generality, we can assume that $x_{0} \in F \backslash E$ : the other case is at all analogous. We then have that

$$
\operatorname{sd}_{F}\left(x_{0}\right)<-c \sqrt{h} .
$$

Then any ball $B_{r}\left(x_{0}\right)$ of radius $r \leq \frac{c \sqrt{h}}{2}$ is contained in $F$. By the minimality of $E$, we have $\mathcal{F}_{h}(E, F) \leq \mathcal{F}_{h}\left(E \cup B_{r}\left(x_{0}\right), F\right)$, and thus

$$
\operatorname{Per}(E) \leq \operatorname{Per}\left(E \cup B_{r}\left(x_{0}\right)\right)+\frac{1}{h} \int_{B_{r}\left(x_{0}\right) \backslash E} \operatorname{sd}_{F} d x+\frac{1}{\sqrt{h}}\left|B_{r}\left(x_{0}\right) \backslash E\right| .
$$

We use (3.5) and $r \leq \frac{c \sqrt{h}}{2}$ to infer that

$$
\frac{1}{h} \int_{B_{r}\left(x_{0}\right) \backslash E} \operatorname{sd}_{F} d x<-\frac{c}{2 \sqrt{h}}\left|B_{r}\left(x_{0}\right) \backslash E\right| .
$$

Then (3.6) and (3.7) yield

$$
\operatorname{Per}(E) \leq \operatorname{Per}\left(E \cup B_{r}\left(x_{0}\right)\right)-h^{-} 1 / 2\left(\frac{c}{2}-1\right)\left|B_{r}\left(x_{0}\right) \backslash E\right| .
$$

By assumption $c>3$ and we can apply Lemma 4.4 with $\mu=0$ and obtain

$$
\left|B_{r}\left(x_{0}\right) \backslash E\right| \geq c_{n} r^{n} \quad \text { for a.e. } 0<r<\frac{c \sqrt{h}}{2} .
$$

On the other hand, from (3.8) we deduce also that for a.e. $0<r<\frac{c \sqrt{h}}{2}$

$$
\begin{aligned}
h^{-1 / 2}\left(\frac{c}{2}-1\right)\left|B_{r}\left(x_{0}\right) \backslash E\right| & \leq \operatorname{Per}\left(E \cup B_{r}\left(x_{0}\right)\right)-\operatorname{Per}(E) \\
& \leq \mathcal{H}^{n-1}\left(\partial B_{r}\left(x_{0}\right) \backslash E\right) \leq n \omega_{n} r^{n-1} .
\end{aligned}
$$

Combining (3.9) and (3.10), we get that

$$
c_{n} r^{n} \leq\left|B_{r}\left(x_{0}\right) \backslash E\right| \leq n \omega_{n}\left(\frac{c}{2}-1\right)^{-1} \sqrt{h} r^{n-1},
$$


for almost all $0<r<\frac{c \sqrt{h}}{2}$, which gives the desired contradiction to the choice of $c$ as soon as $r \uparrow \frac{c \sqrt{h}}{2}$.

The following density estimates are now an immediate consequence.

Corollary 3.3 Let $F \subset \mathbb{R}^{n}$ be a bounded set of finite perimeter and let $E$ be a minimizer of $\mathcal{F}_{h}(\cdot, F)$. Then, for every $r \in\left(0, \gamma_{n} \sqrt{h}\right)$ and for every $x_{0} \in \partial E$, it holds

$$
\begin{array}{r}
\min \left\{\left|B_{r}\left(x_{0}\right) \backslash E\right|,\left|E \cap B_{r}\left(x_{0}\right)\right|\right\} \geq c_{n} r^{n}, \\
c_{n} r^{n-1} \leq \operatorname{Per}\left(E, B_{r}\left(x_{0}\right)\right) \leq C_{n} r^{n-1} .
\end{array}
$$

Proof Since $E$ is a minimizer of $\mathcal{F}_{h}(\cdot, F)$, for any $x_{0} \in \partial E$, it holds that $\mathcal{F}_{h}(E, F) \leq$ $\mathcal{F}_{h}\left(E \backslash B_{r}\left(x_{0}\right), F\right)$, which implies

$$
\operatorname{Per}(E)+\frac{1}{h} \int_{E \cap B_{r}\left(x_{0}\right)} \operatorname{sd}_{F} d x \leq \operatorname{Per}\left(E \backslash B_{r}\left(x_{0}\right)\right)+\frac{1}{\sqrt{h}}\left|E \cap B_{r}\left(x_{0}\right)\right| .
$$

Estimating the second term via Proposition 3.2, we obtain

$$
\operatorname{Per}(E) \leq \operatorname{Per}\left(E \backslash B_{r}\left(x_{0}\right)\right)+\frac{C_{n}}{\sqrt{h}}\left|E \cap B_{r}\left(x_{0}\right)\right| .
$$

A similar analysis shows that

$$
\operatorname{Per}(E) \leq \operatorname{Per}\left(E \cup B_{r}\left(x_{0}\right)\right)+\frac{C_{n}}{\sqrt{h}}\left|B_{r}\left(x_{0}\right) \backslash E\right| .
$$

Therefore, by Lemma 4.4 we deduce (by possibly redefining $c_{n}$ )

$$
\min \left\{\left|E \cap B_{r}\left(x_{0}\right)\right|,\left|B_{r}\left(x_{0}\right) \backslash E\right|\right\} \geq c_{n} r^{n} \quad \forall 0<r \leq \gamma_{n} \sqrt{h} .
$$

The first inequality in (3.12) is now an immediate consequence of the relative isoperimetric inequality (cf. [19, Corollary 1.29]). For the second inequality, we rewrite (3.13) as

$$
\mathcal{H}^{n-1}\left(\partial E \cap B_{r}\left(x_{0}\right)\right) \leq \mathcal{H}^{n-1}\left(\partial B_{r}\left(x_{0}\right) \cap E\right)+\frac{C_{n}}{\sqrt{h}}\left|E \cap B_{r}\left(x_{0}\right)\right| .
$$

Since $r<\gamma_{n} \sqrt{h}$, the upper bound is obvious.

Next we prove an estimate on the volume of the symmetric difference of two consecutive sets of the approximate solutions.

Proposition 3.4 ( $L^{1}$-estimate) Let $F \subset \mathbb{R}^{n}$ be a bounded set of finite perimeter and let $E$ be a minimizer of $\mathcal{F}_{h}(\cdot, F)$. Then,

$$
|E \Delta F| \leq C_{n}\left(\ell \operatorname{Per}(E)+\frac{1}{\ell} \int_{E \Delta F} \mathrm{~d}_{F} d x\right) \forall \ell \leq \gamma_{n} \sqrt{h}
$$

Proof In order to estimate $E \Delta F$, we split it into two parts:

$$
|E \Delta F| \leq\left|\left\{x \in E \Delta F: \mathrm{d}_{F}(x) \leq \ell\right\}\right|+\left|\left\{x \in E \Delta F: \mathrm{d}_{F}(x) \geq \ell\right\}\right| .
$$

The second term is easily estimated by

$$
\left|\left\{x \in E \Delta F: \mathrm{d}_{F}(x) \geq \ell\right\}\right| \leq \frac{1}{\ell} \int_{E \Delta F} \mathrm{~d}_{F}(x) d x .
$$


To estimate the first term, we use a simple covering argument to find a collection of disjoint balls $\left\{B_{\ell}\left(x_{i}\right)\right\}_{i \in I}$ with $x_{i} \in \partial^{*} E$ and $I \subset \mathbb{N}$ a finite set such that $\partial^{*} E \subset \cup_{i \in I} B_{2 \ell}\left(x_{i}\right)$. Note that by (3.11) and the relative isoperimetric inequality (cf. [19, Corollary.1.29]) we have for every $i \in I$

$$
\begin{aligned}
\left|B_{3 \ell}\left(x_{i}\right)\right| & \stackrel{(3.11)}{\leq} C_{n} \min \left\{\left|E \cap B_{\ell}\left(x_{i}\right)\right|,\left|B_{\ell}\left(x_{i}\right) \backslash E\right|\right\} \\
& \leq C_{n} \ell \mathcal{H}^{n-1}\left(\partial^{*} E \cap B_{\ell}\left(x_{i}\right)\right) .
\end{aligned}
$$

Note finally that the set $\left\{x \in E \Delta F: \mathrm{d}_{F}(x) \leq \ell\right\}$ is covered by $\left\{B_{3 \ell}\left(x_{i}\right)\right\}_{i \in I}$. Summing over $i$ and the choice of the balls $\left\{B_{\ell}\left(x_{i}\right)\right\}_{i \in I}$ yields

$$
\begin{aligned}
\left|\left\{x \in E \Delta F: \mathrm{d}_{F}(x) \leq \ell\right\}\right| & \leq \sum_{i \in I}\left|B_{3 \ell}\left(x_{i}\right)\right| \\
& \leq C_{n} \ell \sum_{i \in I} \mathcal{H}^{n-1}\left(\partial^{*} E \cap B_{\ell}\left(x_{i}\right)\right) \\
& \leq C_{n} \ell \operatorname{Per}(E) .
\end{aligned}
$$

\subsection{Hölder continuity in time}

As an immediate consequence of the discrete dissipation inequality (3.2), we remark that

$$
\begin{aligned}
& \operatorname{Per}\left(E_{t}^{(h)}\right)+\frac{1}{h} \int_{E_{t}^{(h)} \Delta E_{t-h}^{(h)}} \mathrm{d}_{E_{t-h}^{(h)}} d x+\frac{1}{\sqrt{h}}|| E_{t}^{(h)}|-1| \\
& \quad \leq \operatorname{Per}\left(E_{t-h}^{(h)}\right)+\frac{1}{\sqrt{h}}|| E_{t-h}^{(h)}|-1| \quad \forall t \in[h,+\infty),
\end{aligned}
$$

and, by iterating (3.15) and using $\left|E_{0}\right|=1$,

$$
\begin{array}{r}
\operatorname{Per}\left(E_{t}^{(h)}\right) \leq \operatorname{Per}\left(E_{0}\right) \quad \forall t \geq 0, \\
\frac{1}{\sqrt{h}}|| E_{t}^{(h)}|-1| \leq \operatorname{Per}\left(E_{0}\right) \quad \forall t \geq 0, \\
\int_{h}^{T} \int_{E_{t}^{(h)} \Delta E_{t-h}^{(h)}} \frac{\mathrm{d}_{E_{t-h}^{(h)}}^{h}}{h} d x \leq \operatorname{Per}\left(E_{0}\right),
\end{array}
$$

for every $T>h$. Similarly, using Proposition 3.4 with $\ell=h<\gamma_{n} \sqrt{h}$, we also get

$$
\begin{aligned}
\int_{h}^{T}\left|E_{t}^{(h)} \Delta E_{t-h}^{(h)}\right| & \leq C_{n} \sum_{k=1}^{[T / h]}\left(h \operatorname{Per}\left(E_{k h}^{(h)}\right)+\int_{E_{k h}^{(h)} \Delta E_{(k-1) h}^{(h)}} \frac{\mathrm{d}_{E_{t-h}^{(h)}}}{h} d x\right) \\
& \leq C_{n}(T+1) \operatorname{Per}\left(E_{0}\right) .
\end{aligned}
$$

Proposition $3.5\left(C^{1 / 2}\right.$ regularity in time) Let $h \leq 1$ and let $\left\{E_{t}\right\}_{t \geq 0}$ be an approximate flat flow. Then it holds

$$
\left|E_{t}^{(h)} \Delta E_{s}^{(h)}\right| \leq C_{n, 0}|t-s|^{1 / 2} \forall 0 \leq t \leq s<+\infty .
$$

Proof Clearly it is enough to consider the case $s-t \geq h$. Let $j \in \mathbb{N}$ and $k \in \mathbb{N} \backslash\{0\}$ be such that $t \in[j h,(j+1) h)$ and $s \in[(j+k) h,(j+k+1) h)$. Then, we can use Proposition 3.4 
with $\ell=\gamma_{n} h /|t-s|^{1} / 2$ (note that $\ell \leq \gamma_{n} \sqrt{h}$ by the assumption $s-t \geq h$ ) and (3.16), and estimate in the following way:

$$
\begin{aligned}
\left|E_{t}^{(h)} \Delta E_{s}^{(h)}\right| \leq & \sum_{m=1}^{k}\left|E_{(j+m) h}^{(h)} \Delta E_{(j+m-1) h}^{(h)}\right| \\
\leq & C_{n} \sum_{m=1}^{k} \frac{h}{|t-s|^{1 / 2}} \operatorname{Per}\left(E_{(j+m) h}^{(h)}\right) \\
& +C_{n} \sum_{m=1}^{k} \frac{|t-s|^{1 / 2}}{h} \int_{E_{(j+m) h}^{(h)} \Delta E_{(j+m-1) h}^{(h)}} \mathrm{d}_{E_{(j+m-1) h}^{(h)}} d x .
\end{aligned}
$$

By using (3.15) we estimate the sum above by

$$
\begin{aligned}
\left|E_{t}^{(h)} \Delta E_{s}^{(h)}\right| \leq & C_{n} \sum_{m=1}^{k} \frac{h}{|t-s|^{1 / 2}} \operatorname{Per}\left(E_{0}\right) \\
& +C_{n} \sum_{m=1}^{k}|t-s|^{1 / 2}\left(\operatorname{Per}\left(E_{(j+m-1) h}^{(h)}\right)-\operatorname{Per}\left(E_{(j+m) h}^{(h)}\right)\right) \\
& +C_{n} \sum_{m=1}^{k} \frac{|t-s|^{1 / 2}}{\sqrt{h}}\left(|| E_{(j+m-1) h}^{(h)}|-1|-|| E_{(j+m) h}^{(h)}|-1|\right) \\
\leq & C_{n} \frac{k h}{|t-s|^{1 / 2}} \operatorname{Per}\left(E_{0}\right)+C_{n}|t-s|^{1 / 2}\left(\operatorname{Per}\left(E_{t}\right)-\operatorname{Per}\left(E_{s}\right)\right) \\
& +C_{n} \frac{|t-s|^{1 / 2}}{\sqrt{h}}\left(|| E_{t}|-1|-|| E_{s}|-1|\right) .
\end{aligned}
$$

Therefore, by (3.16) and (3.17), we get

$$
\left|E_{t}^{(h)} \Delta E_{s}^{(h)}\right| \leq C_{n}|t-s|^{1 / 2} \operatorname{Per}\left(E_{0}\right),
$$

where we used $k h \leq|t-s|+h \leq 2|t-s|$, thus concluding the proof of the proposition.

\subsection{First variations and first consequences}

We now introduce the time-discrete normal velocity: for all $t \geq 0$ and $x \in \mathbb{R}^{n}$, we set

$$
v^{(h)}(t, x):= \begin{cases}\frac{1}{h} \operatorname{sd}_{E_{t-h}^{(h)}}(x) & \text { for } t \in[h,+\infty), \\ 0 & \text { for } t \in[0, h) .\end{cases}
$$

Lemma 3.6 ( $L^{2}$-bound on the velocity) Let $\left\{E_{t}^{(h)}\right\}_{t \geq 0}$ be an approximate flat flow. Then it holds

$$
\int_{0}^{\infty} \int_{\partial E_{t}^{(h)}}\left(v^{(h)}\right)^{2} d \mathcal{H}^{n-1} d t \leq C_{n, 0} .
$$

Proof We fix $t \in[h,+\infty)$ and consider for every $\ell \in \mathbb{Z}$ with $2^{\ell} \leq \gamma_{n} / \sqrt{h}$ the sets

$$
K(\ell):=\left\{x \in \mathbb{R}^{d}: 2^{\ell}<\left|v^{(h)}(t, x)\right| \leq 2^{\ell+1}\right\},
$$


so that $\mathbb{R}^{n}=\bigcup_{\ell} K(\ell)$. It follows from $2^{\ell-1} h \leq \gamma_{n} \sqrt{h} / 2$ and from Corollary 3.3 that, for every $x \in \partial E_{t}^{(h)}$,

$$
\begin{aligned}
& \left|E_{t}^{(h)} \cap B_{2^{\ell-1} h}(x)\right| \geq c_{n}\left(2^{\ell-1} h\right)^{n}, \\
& \mathcal{H}^{n-1}\left(\partial E_{t}^{(h)} \cap B_{2^{\ell-1} h}(x)\right) \leq C_{n}\left(2^{\ell-1} h\right)^{n-1} .
\end{aligned}
$$

Using $2^{\ell-1} \leq\left|v^{(h)}(t, y)\right| \leq 4 \cdot 2^{\ell-1}$ for all $y \in B_{2^{\ell-1} h}(x)$ with $x \in \partial E_{t}^{(h)} \cap K(\ell)$, we obtain for every $x \in \partial E_{t}^{(h)} \cap K(\ell)$

$$
\begin{aligned}
& \int_{B_{2^{\ell-1} h}(x) \cap\left(E_{t}^{(h)} \Delta E_{t-h}^{(h)}\right)}\left|v^{(h)}\right| d y \stackrel{(3.23)}{\geq} c_{n} 2^{\ell-1}\left(2^{\ell-1} h\right)^{n}, \\
& \int_{B_{2^{\ell-1} h}(x) \cap \partial E_{t}^{(h)}}\left(v^{(h)}\right)^{2} d \mathcal{H}^{n-1} \stackrel{(3.24)}{\leq} C_{n}\left(2^{\ell-1}\right)^{2}\left(2^{\ell-1} h\right)^{n-1} .
\end{aligned}
$$

Hence, combining these two estimates, we have

$$
\int_{B_{2^{\ell-1} h}(x) \cap \partial E_{t}^{(h)}}\left(v^{(h)}\right)^{2} d \mathcal{H}^{n-1} \leq \frac{C_{n}}{h} \int_{B_{2^{\ell-1} h}(x) \cap\left(E_{t}^{(h)} \Delta E_{(k-1) h}^{(h)}\right)}\left|v^{(h)}\right| d y .
$$

Now, by a simple application of Besicovitch's covering theorem [16, Chapter 1.5.2] to $\left\{B_{2^{\ell-1} h}(x): x \in \partial E_{t}^{(h)} \cap K(\ell)\right\}$, we obtain

$$
\int_{\partial E_{t}^{(h)} \cap K(\ell)}\left(v^{(h)}\right)^{2} d \mathcal{H}^{n-1} \leq \frac{C_{n}}{h} \int_{\left(E_{t}^{(h)} \Delta E_{t-h}^{(h)}\right) \cap\left\{2^{\ell-2} \leq\left|v^{(h)}\right| \leq 2^{\ell+2}\right\}}\left|v^{(h)}\right| d x .
$$

Finally, summing up over $\ell \in \mathbb{Z}$ with $2^{\ell} \leq \frac{\gamma_{n}}{\sqrt{h}}$ in (3.25) yields

$$
\int_{\partial E_{t}^{(h)}}\left(v^{(h)}\right)^{2} d \mathcal{H}^{n-1} \leq \frac{C_{n}}{h} \int_{E_{t}^{(h)} \Delta E_{t-h}^{(h)}}\left|v^{(h)}\right| d x .
$$

We now show how the above estimate implies (3.22). In view of (3.15) we have

$$
\begin{aligned}
& \int_{\partial E_{t}^{(h)}}\left(v^{(h)}\right)^{2} d \mathcal{H}^{n-1} \\
& \quad \leq \frac{C_{n}}{h}\left(\operatorname{Per}\left(E_{t-h}^{(h)}\right)+\frac{1}{\sqrt{h}}|| E_{t-h}^{(h)}|-1|-\operatorname{Per}\left(E_{t}^{(h)}\right)-\frac{1}{\sqrt{h}}|| E_{t}^{(h)}|-1|\right) .
\end{aligned}
$$

Integrating in time and using $\left|E_{0}\right|=1$, we obtain

$$
\begin{aligned}
& \int_{0}^{T} \int_{\partial E_{t}^{(h)}}\left(v^{(h)}\right)^{2} d \mathcal{H}^{n-1} d t \\
& \leq C_{n}\left(\operatorname{Per}\left(E_{0}^{(h)}\right)-\operatorname{Per}\left(E_{T}^{(h)}\right)-\frac{1}{\sqrt{h}}|| E_{T}^{(h)}|-1|\right) \\
& \leq C_{n} \operatorname{Per}\left(E_{0}\right),
\end{aligned}
$$

from which, by a simple limit for $T \rightarrow+\infty$, (3.22) follows.

We now derive the Euler-Lagrange equations which constitute the weak motion law for the time-discrete evolution. 
Lemma 3.7 (Euler-Lagrange equations) For every $t \in[h,+\infty)$ and $\Psi \in C_{c}^{1}\left(\mathbb{R}^{n} ; \mathbb{R}^{n}\right)$, it holds

$$
\int_{\partial E_{t}^{(h)}}\left(\operatorname{div}_{\partial E_{t}^{(h)}} \Psi+v^{(h)} v_{E_{t}^{(h)}} \cdot \Psi\right) d \mathcal{H}^{n-1}=\lambda^{(h)}(t) \int_{\partial E_{t}^{(h)}} v_{E_{t}^{(h)}} \cdot \Psi d \mathcal{H}^{n-1},
$$

where

$$
\lambda^{(h)}(t):=\frac{1}{\mathcal{H}^{n-1}\left(\partial E_{t}^{(h)}\right)} \int_{\partial E_{t}^{(h)}}\left(H_{E_{t}^{(h)}}+v^{(h)}\right) d \mathcal{H}^{n-1} .
$$

Moreover, if $\left|E_{t}^{(h)}\right| \neq 1$, then it also holds $\lambda^{(h)}(t)=\frac{1}{\sqrt{h}} \operatorname{sgn}\left(1-\left|E_{t}^{(h)}\right|\right)$.

As we shall see in the proof below, the constants $\lambda^{(h)}(t)$ defined in (3.27) are Lagrange multipliers corresponding to the volume constraint, whenever it is active. Since this constraint is satisfied up to a finite number of times (uniformly in $h$ ) by Corollary 3.10 below, by a slight abuse of terminology, we call $\lambda^{(h)}(t)$ a Lagrange multiplier, even if the volume constraint is not active.

Proof If $\left|E_{t}^{(h)}\right| \neq 1$, it is very simple to compute the variations of $\mathcal{F}_{h}\left(\cdot, E_{t-h}^{(h)}\right)$ along the vector field $\Psi \in C_{c}^{1}\left(\mathbb{R}^{n} ; \mathbb{R}^{n}\right)$ and see that they are given by (3.26) with $\lambda^{(h)}(t)=\frac{1}{\sqrt{h}} \operatorname{sgn}\left(1-\left|E_{t}^{(h)}\right|\right)$. In the case $\left|E_{t}^{(h)}\right|=1$, we have

$$
E_{t}^{(h)} \in \arg \min \left\{\operatorname{Per}(F)+\int_{F \Delta E_{t-h}^{(h)}} \mathrm{d}_{E_{t-h}^{(h)}} d x:|F|=1\right\} .
$$

Hence, performing variations of

$$
\operatorname{Per}(F)+\int_{F \Delta E_{t-h}^{(h)}} \mathrm{d}_{E_{t-h}^{(h)}} d x
$$

within the class of sets of unit volume, for every $\Psi \in C_{c}^{1}\left(\mathbb{R}^{n} ; \mathbb{R}^{n}\right)$, we again find (3.26), where $\lambda^{(h)}(t)$ is the Lagrange multiplier related to the constraint $|F|=1$. Observe that in both cases, we can choose a sequence of $\Psi \in C_{c}^{1}\left(\mathbb{R}^{n} ; \mathbb{R}^{n}\right)$ approximating $v_{E_{t}^{(h)}}$ on $\partial E_{t}^{(h)}$, and conclude that $\lambda^{(h)}(t)$ is given by (3.27).

It is now clear that the regular part of $\partial^{*} E_{t}^{(h)}$ is of class $C^{2, \kappa}$. Indeed, by choosing a suitable system of coordinates, $\partial^{*} E_{t}^{(h)}$ can be written in a neighbourhood of any regular point as the graph of a $C^{1, \kappa}$ function $u$ solving the following equation in the sense of distributions:

$$
\operatorname{div}\left(\frac{\nabla u}{\sqrt{1+|\nabla u|^{2}}}\right)=v^{(h)}-\lambda^{(h)} \text {. }
$$

Since $v^{(h)}$ is Lipschitz continuous, by standard elliptic regularity theory (cp., e.g., [18]), one then deduce that $u \in C^{2, \kappa}$ for every $\kappa \in(0,1)$.

Next we prove that the whole family of sets defining the discrete flow up to time $T>0$ is contained in a large ball, whose radius does not depend on the discrete time-step $h$ but may depend on the $T$.

Lemma 3.8 (Boundedness of minimizing sets) Let $\left\{E_{t}^{(h)}\right\}_{t \geq 0}$ be an approximate solution and let $T>0$. Then there exist $h_{0}, R_{T}>0$ (depending on $T, n$, and $E_{0}$ only) such that, if $h \leq h_{0}$, then $E_{t}^{(h)} \subset B_{R_{T}}$ for all $t \in[0, T]$. 
Proof We fix $h>0$, and for every $t \in[0, T)$ we let

$$
r_{t}:=\inf \left\{r>0: E_{t}^{(h)} \subset B_{r}\right\} .
$$

We notice $\bar{B}_{r_{t}} \cap \partial E_{t}^{(h)} \neq \emptyset$ is made of regular points (because there are no singular minimizing cones contained in a half space, cp. [19, Theorem 15.5]), and moreover

$$
\bar{B}_{r_{t}} \cap \partial E_{t}^{(h)} \subset\left\{y \in \partial E_{t}^{(h)}: H_{E_{t}^{(h)}}(y) \geq 0\right\} .
$$

By this observation and the Euler-Lagrange equation $v^{(h)}(t, y)=\lambda^{(h)}(t)-H_{E_{t}^{(h)}}(y)$, it follows that

$$
r_{t} \leq r_{t-h}+h\left|\lambda^{(h)}(t)\right| .
$$

Iterating the above estimate, we then deduce that

$$
r_{\tau} \leq r_{0}+\int_{0}^{\tau}\left|\lambda^{(h)}(t)\right| d t \quad \forall \tau \in[0, T]
$$

To get some control on $\lambda^{(h)}(t)$ we consider $\Psi \in C_{c}^{1}\left(\mathbb{R}^{n} ; \mathbb{R}^{n}\right)$ such that $\Psi(x)=x$ in $B_{r_{t}}$, and, since for $h$ small enough $\left|E_{t}^{(h)}\right| \geq \frac{1}{2}$ by (3.17), using $\Psi$ as test in (3.26), we obtain

$$
\begin{aligned}
\frac{n}{2}\left|\lambda^{(h)}(t)\right| & \leq\left|\lambda^{(h)}(t) \int_{E_{t}^{(h)}} \operatorname{div} \Psi d x\right|=\left|\lambda^{(h)}(t) \int_{\partial E_{t}^{(h)}} v_{E_{t}^{(h)}} \cdot \Psi d \mathcal{H}^{n-1}\right| \\
& =\left|\int_{\partial E_{t}^{(h)}}\left(\operatorname{div}_{\partial E_{t}^{(h)}} \Psi+v^{(h)} v_{E_{t}^{(h)}} \cdot \Psi\right) d \mathcal{H}^{n-1}\right| \\
& \leq(n-1) \operatorname{Per}\left(E_{t}^{(h)}\right)+r_{t} \operatorname{Per}\left(E_{t}^{(h)}\right)^{1 / 2}\left\|v^{(h)}(t, \cdot)\right\|_{L^{2}\left(\partial E_{t}^{(h)}\right)}
\end{aligned}
$$

where we used $|\Psi| \leq r_{t}$ on $\partial E_{t}^{(h)}$. Integrating in time and using the Cauchy-Schwarz inequality together with (3.16) and (3.22), we obtain

$$
\int_{0}^{\tau}\left|\lambda^{(h)}(t)\right| d t \leq C_{n, 0} \tau+C_{n, 0}\left(\int_{0}^{\tau} r_{t}^{2} d t\right)^{1 / 2} .
$$

Combining (3.28) and (3.30), it follows that

$$
r_{\tau} \leq r_{0}+C_{n, 0} \tau+C_{n, 0}\left(\int_{0}^{\tau} r_{t}^{2} d t\right)^{1 / 2} \text { for all } \tau \in[0, T] .
$$

The remainder of the proof is a standard ODE argument. Indeed, squaring both sides of the equation and redefining $C_{n, 0}$ yields

$$
\frac{d}{d \tau}\left(e^{-C_{n, 0} \tau} F(\tau)\right) \leq C_{n, 0} e^{-C_{n, 0} \tau}\left(r_{0}^{2}+\tau^{2}\right) \text { for all } \tau \in[0, T]
$$

where $F(\tau)=\int_{0}^{\tau} r_{t}^{2} d t$. Integration in $\tau$ over the interval $[0, T]$ yields

$$
\int_{0}^{T} r_{t}^{2} d t \leq C_{n, 0, T}
$$

and thus the statement in Lemma 3.8 follows via (3.31). 
Corollary 3.9 For every $h>0$ small enough, it holds

$$
\int_{0}^{T} \int_{\partial E_{t}^{(h)}} H_{E_{t}^{(h)}}^{2} d \mathcal{H}^{n-1} d t+\int_{0}^{T}\left|\lambda^{(h)}(t)\right|^{2} d t \leq C_{n, 0, T} .
$$

Proof The integrability of $\lambda^{(h)}$ follows from (3.29) and from Lemma 3.8; the one of $H_{E_{t}^{(h)}}$ follows taking into account the first variation (3.26) and the integrability of the discrete velocity, Lemma 3.6.

For every $h>0$ we set

$$
\Sigma(h):=\left\{t:\left|E_{t}^{(h)}\right| \neq 1\right\} .
$$

Corollary 3.10 For every $h>0$ small enough, we have

$$
|\Sigma(h)| \leq C_{n, 0, T} h .
$$

Proof In view of Lemma 3.7, it is

$$
\Sigma(h) \subset\left\{t \in[0, T):\left|\lambda^{(h)}(t)\right| \geq 1 / \sqrt{h}\right\},
$$

and thus we have by Corollary 3.9

$$
|\Sigma(h)| \leq h \int_{0}^{T}\left|\lambda^{(h)}(t)\right|^{2} d t \leq C_{n, 0, T} h
$$

\subsection{Proof of Theorem 2.2}

The proof of the existence of a flat flow is now a simple consequence of the results above. Indeed, by (3.16), (3.17) and Lemma 3.8, one can find sets $\left\{E_{t}\right\}_{t \in \mathbb{Q}^{+}}$, where $\mathbb{Q}^{+}$denotes the set of positive rational numbers, and a subsequence $h_{k} \downarrow 0$ such that

$$
\lim _{k \rightarrow+\infty}\left|E_{t}^{\left(h_{k}\right)} \Delta E_{t}\right|=0 \quad \forall t \in \mathbb{Q}^{+} .
$$

Using the triangular inequality and Proposition 3.5, we deduce that

$$
\begin{aligned}
\left|E_{t} \Delta E_{s}\right| & \leq \lim _{k \rightarrow+\infty}\left(\left|E_{t} \Delta E_{t}^{\left(h_{k}\right)}\right|+\left|E_{t}^{\left(h_{k}\right)} \Delta E_{s}^{\left(h_{k}\right)}\right|+\left|E_{s}^{\left(h_{k}\right)} \Delta E_{s}\right|\right) \\
& \leq C_{n, 0}|s-t|^{1 / 2} \quad \forall 0 \leq s \leq t \in \mathbb{Q}^{+} .
\end{aligned}
$$

Now a simple continuity argument implies that the sequence $E_{t}^{\left(h_{k}\right)}$ converges to sets $E_{t}$ for all times $t \geq 0$ and satisfies (3.32) for all $s, t \in[0,+\infty)$. Finally, note that passing to the limit in (3.15) yields that $\left|E_{t}\right|=1$ and $\operatorname{Per}\left(E_{t}\right) \leq \operatorname{Per}\left(E_{0}\right)$ (cf. [16, Sect 5.2.1]) for a.e. $t \in(0, T)$.

Remark 3.11 It is also possible to show that the sequence of characteristic functions

$$
\chi^{(h)}(t, x):=\chi_{E_{t}^{(h)}}(x)
$$

are precompact in $L^{1}\left((0, T) \times \mathbb{R}^{n}\right)$ for every $T>0$, thus giving an alternative proof of the theorem. 


\section{Distributional solutions}

In this section we prove Theorem 2.3 on the existence of distributional solutions. The two main ingredients of the proof besides the estimates of the previous section are the hypothesis (2.2) on the continuity of the perimeters of the approximate solutions and the following proposition which links the discrete velocity to the distributional time derivative of the flat flow.

Proposition 4.1 Let $n \leq 7$ and $\left\{E_{t}^{(h)}\right\}_{t \geq 0}$ be an approximate solution to the volumepreserving mean-curvature flow. Then, for every $\phi \in C_{c}^{\infty}\left([0,+\infty) \times \mathbb{R}^{n}\right)$ it holds

$$
\lim _{h \rightarrow 0}\left|\int_{h}^{+\infty} \frac{1}{2 h}\left[\int_{E_{t}^{(h)}} \phi d x-\int_{E_{t-h}^{(h)}} \phi d x\right] d t-\int_{h}^{+\infty} \int_{\partial E_{t}^{(h)}} \phi v_{h} d \mathcal{H}^{n-1} d t\right|=0 .
$$

Assuming the proposition we give a proof of the theorem.

\subsection{Proof of Theorem 2.3}

It follows straightforwardly from (2.2) (cp., for instance, [3, Proposition 1.80]) that $\mathcal{H}^{n-1} \partial^{*} E_{t}^{\left(h_{k}\right)}$ weakly converges to $\mathcal{H}^{n-1} \partial^{*} E_{t}$ for almost every $t \in[0,+\infty)$. In particular this implies that, for almost every $t \in[0,+\infty)$, the boundaries $\partial^{*} E_{t}^{\left(h_{k}\right)}$ converge to $\partial^{*} E_{t}$ in the sense of varifolds: namely, for a.e. $t \in[0,+\infty)$ it holds

$$
\lim _{k \rightarrow \infty} \int_{\partial^{*} E_{t}^{\left(h_{k}\right)}} F\left(x, v_{E_{t}^{\left(h_{k}\right)}}(x)\right) d \mathcal{H}^{n-1}(x)=\int_{\partial^{*} E_{t}} F\left(x, v_{E_{t}}(x)\right) d \mathcal{H}^{n-1}(x),
$$

for every $F \in C_{c}\left(\mathbb{R}^{n} \times \mathbb{R}^{n}\right)$. Indeed, by a simple approximation argument it is easy to verify that it is enough to consider $F \in C_{c}^{1}\left(\mathbb{R}^{n} \times \mathbb{R}^{n}\right)$. Then, for every $\varepsilon>0$ we pick a continuous function $v_{\varepsilon}: \mathbb{R}^{n} \rightarrow \mathbb{R}^{n}$ such that

$$
\int_{\partial^{*} E_{t}}\left|v_{E_{t}}-v_{\varepsilon}\right|^{2} d \mathcal{H}^{n-1} \leq \varepsilon^{2}
$$

and estimate as follows

$$
\begin{aligned}
& \lim _{k \rightarrow+\infty}\left|\int_{\partial^{*} E_{t}^{\left(h_{k}\right)}}\left(F\left(x, v_{E_{t}^{\left(h_{k}\right)}}(x)\right)-F\left(x, v_{\varepsilon}(x)\right)\right) d \mathcal{H}^{n-1}(x)\right| \\
& \leq \lim _{k \rightarrow+\infty}\|D F\|_{L^{\infty}} \int_{\partial^{*} E_{t}^{\left(h_{k}\right)}} \mid v_{E_{t}^{\left(h_{k}\right)}-v_{\varepsilon} \mid d \mathcal{H}^{n-1}} \\
& \leq \lim _{k \rightarrow+\infty}\|D F\|_{L^{\infty}} \operatorname{Per}\left(E_{t}^{\left(h_{k}\right)}\right)^{1 / 2}\left(\int_{\partial^{*} E_{t}^{\left(h_{k}\right)}}\left|v_{E_{t}^{\left(h_{k}\right)}}-v_{\varepsilon}\right|^{2} d \mathcal{H}^{n-1}\right)^{1 / 2} \\
& =\|D F\|_{L^{\infty}} \operatorname{Per}\left(E_{t}\right)^{1 / 2}\left(\int_{\partial^{*} E_{t}}\left|v_{E_{t}}-v_{\varepsilon}\right|^{2} d \mathcal{H}^{n-1}\right)^{1 / 2} \\
& \leq\|D F\|_{L^{\infty}} \operatorname{Per}\left(E_{t}\right)^{1 / 2} \varepsilon,
\end{aligned}
$$

where we used (2.2) and the weak convergence of the the vector valued measures $v_{E_{t}}^{\left(h_{k}\right)} \mathcal{H}^{n-1} \partial^{*} E_{t}^{\left(h_{k}\right)} \stackrel{*}{\rightarrow} v_{E_{t}} \mathcal{H}^{n-1} \partial^{*} E_{t}$ in the following way: 


$$
\begin{aligned}
\lim _{k \rightarrow+\infty} \int_{\partial^{*} E_{t}^{\left(h_{k}\right)}} \mid v_{E_{t}^{\left(h_{k}\right)}-\left.v_{\varepsilon}\right|^{2} d \mathcal{H}^{n-1}} & =\lim _{k \rightarrow+\infty} \int_{\partial^{*} E_{t}^{\left(h_{k}\right)}}\left(1+\left|v_{\varepsilon}\right|^{2}-2 v_{\left.E_{t}^{\left(h_{k}\right)} \cdot v_{\varepsilon}\right) d \mathcal{H}^{n-1}}\right. \\
& =\int_{\partial^{*} E_{t}}\left(1+\left|v_{\varepsilon}\right|^{2}-2 v_{E_{t}} \cdot v_{\varepsilon}\right) d \mathcal{H}^{n-1} \\
& =\int_{\partial^{*} E_{t}}\left|v_{E_{t}}-v_{\varepsilon}\right|^{2} d \mathcal{H}^{n-1} .
\end{aligned}
$$

Next we use Lemma 3.6 and Corollary 3.9 in conjunction with the results in Hutchinson [23, Theorem 4.4.2] to deduce the existence of functions $v:[0,+\infty) \times \mathbb{R}^{n} \rightarrow \mathbb{R}, \widehat{\lambda}$ : $[0,+\infty) \rightarrow \mathbb{R}$ and $H:[0,+\infty) \times \mathbb{R}^{n} \rightarrow \mathbb{R}$ such that

$$
\int_{0}^{T}|\widehat{\lambda}|^{2} d t+\int_{0}^{T} \int_{\partial^{*} E_{t}}\left(|v|^{2}+|H|^{2}\right) \mathcal{H}^{n-1} d t<C_{n, 0, T}
$$

and

$$
\begin{aligned}
& \lim _{k \rightarrow \infty} \int_{0}^{T} \int_{\partial E_{t}^{\left(h_{k}\right)}} v_{h_{k}} \phi d \mathcal{H}^{n-1} d t=\int_{0}^{T} \int_{\partial^{*} E_{t}} v \phi d \mathcal{H}^{n-1} d t, \\
& \lim _{k \rightarrow \infty} \int_{0}^{T} \int_{\partial E_{t}^{\left(h_{k}\right)}} \lambda^{\left(h_{k}\right)} \phi d \mathcal{H}^{n-1} d t=\int_{0}^{T} \int_{\partial^{*} E_{t}} \widehat{\lambda} \phi d \mathcal{H}^{n-1} d t, \\
& \lim _{k \rightarrow \infty} \int_{0}^{T} \int_{\partial E^{\left(h_{k}\right)}} H_{E_{t}^{\left(h_{k}\right)}} v_{E^{\left(h_{k}\right)}} \cdot \Phi d \mathcal{H}^{n-1} d t=\int_{0}^{T} \int_{\partial^{*} E_{t}} H \cdot \Phi d \mathcal{H}^{n-1} d t,
\end{aligned}
$$

for every $\phi \in C_{c}^{0}\left([0, T) \times \mathbb{R}^{n}\right)$ and every $\Phi \in C_{c}^{0}\left([0, T) \times \mathbb{R}^{n} ; \mathbb{R}^{n}\right)$.

In particular, testing (4.2) with $F(x, v):=\operatorname{div} \Psi-v \cdot \nabla \Psi v$ for some $\Psi \in C_{c}^{1}\left(\mathbb{R}^{n} ; \mathbb{R}^{n}\right)$ and using (4.5), by a simple approximation argument we conclude that, for a.e. $t \in[0,+\infty)$,

$$
\begin{aligned}
\int_{\partial^{*} E_{t}} \operatorname{div}_{\partial E^{t}} \Psi d \mathcal{H}^{n-1} & =\lim _{k \rightarrow+\infty} \int_{\partial^{*} E_{t}^{\left(h_{k}\right)}} \operatorname{div}_{\partial E_{t}^{\left(h_{k}\right)} \Psi d \mathcal{H}^{n-1}} \\
& =\lim _{k \rightarrow+\infty} \int_{\partial^{*} E_{t}^{\left(h_{k}\right)}} v_{E_{t}^{\left(h_{k}\right)} \cdot \Psi H_{E_{t}^{\left(h_{k}\right)}} d \mathcal{H}^{n-1}} \\
& =\int_{\partial^{*} E_{t}} v_{E_{t}} \cdot \Psi H d \mathcal{H}^{n-1},
\end{aligned}
$$

thus showing that $H(t, \cdot)$ is the generalized mean-curvature of $E_{t}$ for a.e. $t \in[0,+\infty)$ (cp. (2.1)) and proving (2.3) of Theorem 2.3.

Similarly, (2.4) and (2.6) follows from (3.26) and (3.27) by using (4.3) and (4.4).

We need only to show (2.5). To this aim we use Proposition 4.1. For every $\phi \in$ $C_{c}^{1}\left([0,+\infty) \times \mathbb{R}^{n}\right)$, by a change of variables we have that

$\int_{h}^{\infty}\left[\int_{E_{t}^{(h)}} \phi d x-\int_{E_{t-h}^{(h)}} \phi d x\right] d t=\int_{h}^{\infty} \int_{E_{t}^{(h)}}(\phi(t, x)-\phi(t+h, x)) d x d t-h \int_{E_{0}} \phi d x$,

where we used that $E_{t}^{(h)}=E_{0}$ for $t \in[0, h)$. Therefore it follows by a simple convergence argument that

$$
\lim _{h \rightarrow 0} \int_{h}^{\infty} \frac{1}{h}\left[\int_{E_{t}^{(h)}} \phi d x-\int_{E_{t-h}^{(h)}} \phi d x\right] d t=-\int_{0}^{\infty} \int_{E_{t}} \frac{\partial \phi}{\partial t}(t, x) d x d t-\int_{E_{0}} \phi d x .
$$


In view of (4.3) and (4.1), we conclude (2.5) straightforwardly.

\subsection{Tilting of the tangent planes}

In this subsection and in the next one we give the proof of Proposition 4.1. We follow closely the arguments in [26] and for the sake of completeness we provide a detailed proof in different steps.

This subsection is devoted to the estimate of the tilting of the normals around points of small curvature. We recall that we assume in this section $n \leq 7$ (in particular, the approximate solutions of the volume-preserving mean-curvature flow are everywhere of class $C^{2, \kappa}$ ).

Lemma 4.2 For given constants $\frac{1}{2}<\beta<\alpha<1$, there exists a continuous increasing function $\omega:[0,1] \rightarrow \mathbb{R}$ with $\omega(0)=0$ with the following property. Let $t \in[2 h,+\infty)$, $\left\{E_{t}^{(h)}\right\}_{t \geq 0}$ be an approximate solution to the volume-preserving mean-curvature flow, and let $x_{0} \in \partial E_{t}^{(h)}$ be such that

$$
\left|v^{(h)}(t, y)\right| \leq h^{\alpha-1} \quad \forall y \in B_{\gamma_{n} \sqrt{h}}\left(x_{0}\right) \cap\left(E_{t}^{(h)} \Delta E_{t-h}^{(h)}\right) .
$$

Then there exists $v \in \mathbb{R}^{n}$ such that $|\nu|=1$ and

$$
\begin{gathered}
\left|v_{\partial E_{t}^{(h)}}(y)-v\right| \leq \omega(h) \quad \forall y \in B_{h^{\beta}}\left(x_{0}\right) \cap \partial E_{t}^{(h)}, \\
\left|v_{\partial E_{t-h}^{(h)}}(y)-v\right| \leq \omega(h) \quad \forall y \in B_{h^{\beta}}\left(x_{0}\right) \cap \partial E_{t-h}^{(h)} .
\end{gathered}
$$

Proof Let $0<R \leq h^{\frac{1}{2}-\beta}$ and let $F \subset \mathbb{R}^{n}$ be any set such that $E_{t}^{(h)} \Delta F \subset \subset B_{R h^{\beta}}\left(x_{0}\right)$. By the minimizing property of $E_{t}^{(h)}$ we have that

$$
\begin{aligned}
\operatorname{Per}\left(E_{t}^{(h)}, B_{R h^{\beta}}\left(x_{0}\right)\right) \leq & \operatorname{Per}\left(F, B_{R h^{\beta}}\left(x_{0}\right)\right)+\frac{1}{h} \int_{F \Delta E_{t}^{(h)}} \mathrm{d}_{E_{t-h}^{(h)}}(y) d y \\
& +\frac{1}{\sqrt{h}}\left(|| F|-1|-|| E_{t}^{(h)}|-1|\right) .
\end{aligned}
$$

A straightforward computation yields

$$
\begin{array}{r}
|| F|-1|-|| E_{t}^{(h)}|-1| \leq\left|F \Delta E_{t}^{(h)}\right|, \\
\frac{1}{h} \int_{F \Delta E_{t}^{(h)}} \mathrm{d}_{E_{t-h}^{(h)}}(y) d y \leq \frac{\gamma_{n}+1}{\sqrt{h}}\left|F \Delta E_{t}^{(h)}\right|,
\end{array}
$$

where we used that $\left|v^{(h)}(t, y)\right| \leq R h^{\beta-1}+\gamma_{n} h^{-1 / 2} \leq\left(\gamma_{n}+1\right) h^{-\frac{1}{2}}$ for all $y \in B_{R h^{\beta}}\left(x_{0}\right) \cap$ $\left(E_{t}^{(h)} \Delta F\right)$ thanks to the fact that $x_{0} \in \partial E_{t}^{(h)}$, Proposition 3.2 and the 1-Lipschitz continuity of the signed distance $\operatorname{sd}_{E_{t-h}^{(h)}}$. Combining the above estimates with (4.9), we obtain

$$
\operatorname{Per}\left(E_{t}^{(h)}, B_{R h^{\beta}}\left(x_{0}\right)\right) \leq \operatorname{Per}\left(F, B_{R h^{\beta}}\left(x_{0}\right)\right)+\frac{\gamma_{n}+2}{\sqrt{h}}\left|F \Delta E_{t}^{(h)}\right| .
$$

Next we introduce the sets

$$
\begin{aligned}
& E_{t}^{(h), \beta}:=\left\{z \in \mathbb{R}^{n}: z=\frac{y-x_{0}}{h^{\beta}}, y \in E_{t}^{(h)}\right\}, \\
& E_{t-h}^{(h), \beta}:=\left\{z \in \mathbb{R}^{n}: z=\frac{y-x_{0}}{h^{\beta}}, y \in E_{t-h}^{(h)}\right\} .
\end{aligned}
$$


By a simple rescaling argument, from (4.10) and from the analogous estimates at time $t-h$ (recall that $t \geq 2 h$ ) we deduce that for $s=t, t-h$

$$
\begin{array}{r}
\operatorname{Per}\left(E_{s}^{(h), \beta}, B_{R}\right) \leq \operatorname{Per}\left(F, B_{R}\right)+\left(\gamma_{n}+2\right) h^{\beta-\frac{1}{2}}\left|F \Delta E_{s}^{(h), \beta}\right| \\
\forall R \leq h^{1 / 2-\beta}, \quad \forall F \Delta E_{s}^{(h), \beta} \subset \subset B_{R} .
\end{array}
$$

This implies that $E_{t}^{(h), \beta}$ and $E_{t-h}^{(h), \beta}$ are both $\left(\Lambda_{h}, r_{h}\right)$-minimizers of the perimeter on $\Lambda_{h}:=$ $\left(\gamma_{n}+2\right) h^{\beta-\frac{1}{2}}$ and $r_{h}:=h^{\frac{1}{2}-\beta}$. By the precompactness for sequences of $\Lambda_{h}$-minimizers (cf. [27, Proposition 21.13]), we conclude that we can find a subsequence (not relabeled) verifying

$$
\lim _{h \rightarrow 0} \chi_{E_{t}^{(h), \beta}}=\chi_{E_{1}^{\beta}} \quad \text { and } \quad \lim _{h \rightarrow 0} \chi_{E_{t-h}^{(h), \beta}}=\chi_{E_{2}^{\beta}} \quad \text { in } L_{\text {loc }}^{1}\left(\mathbb{R}^{n}\right) .
$$

Moreover, using the lower semicontinuity of the perimeter with respect to $L^{1}$ convergence and $\beta>\frac{1}{2}$, we deduce that $E_{1}^{\beta}$ and $E_{2}^{\beta}$ are locally minimizing the perimeter. By the assumption $n \leq 7$ and a Bernstein theorem (see [19, Theorem 17.3]), $E_{1}^{\beta}, E_{2}^{\beta}$ are half-spaces. Moreover, by hypothesis it holds

$$
\mathrm{d}_{E_{t-h}^{(h), \beta}}(z) \leq h^{\alpha-\beta} \quad \forall z \in B_{h^{\frac{1}{2}-\beta}}(0) \cap\left(E_{t}^{(h), \beta} \Delta E_{t-h}^{(h), \beta}\right),
$$

thus implying that $E_{1}^{\beta}=E_{2}^{\beta}$, and by the fact that both are hyperplanes there exists $v \in \mathbb{R}^{n}$ with $|v|=1$ such that

$$
E_{1}^{\beta}=E_{2}^{\beta}=\left\{z \in \mathbb{R}^{n}: z \cdot v<0\right\} .
$$

To reach the conclusion of the lemma we need only to invoke the regularity theory of $\Lambda$-minimizing set (cp. [27, Theorem 26.3]) and conclude that $\partial E_{s}^{(h), \beta}$ is uniformly $C^{1, \kappa}$ in $B_{1}$ for $s=t, t-h$, thus leading straightforwardly to (4.7) and (4.8).

Corollary 4.3 Under the hypotheses of Lemma 4.2, let $\mathbf{C}_{h^{\beta} / 2}\left(x_{0}, v\right) \subset \mathbb{R}^{n}$ be the open cylinder defined as

$$
\begin{aligned}
& \mathbf{C}_{h^{\beta} / 2}\left(x_{0}, v\right) \\
& :=\left\{x \in \mathbb{R}^{n}:\left|\left(x-x_{0}\right) \cdot v\right|<h^{\beta} / 2, \sqrt{\left|x-x_{0}\right|^{2}-\left|\left(x-x_{0}\right) \cdot v\right|^{2}}<h^{\beta} / 2\right\} .
\end{aligned}
$$

Then, there exists a dimensional constant $C>0$ such that

$$
\begin{aligned}
& \left|\int_{\mathbf{C}_{h^{\beta} / 2}\left(x_{0}, v\right)}\left(\chi_{E_{t}^{(h)}}-\chi_{E_{t-h}^{(h)}}\right) d x-\int_{\partial E_{t}^{(h)} \cap \mathbf{C}_{h^{\beta} / 2}\left(x_{0}, v\right)} \operatorname{sd}_{E_{t-h}^{(h)}} d \mathcal{H}^{n-1}\right| \\
& \quad \leq C \omega(h) \int_{\mathbf{C}_{h^{\beta} / 2}\left(x_{0}, v\right)}\left|\chi_{E_{t}^{(h)}}-\chi_{E_{t-h}^{(h)}}\right| d x .
\end{aligned}
$$

Proof From Lemma 4.2 we know that, for $h$ sufficiently small, $\partial E_{t}^{(h)}$ and $\partial E_{t-h}^{(h)}$ in $\mathbf{C}_{h^{\beta} / 2}\left(x_{0}, v\right)$ can both be written as graphs of functions of class $C^{1, \kappa}$. Namely, by an affine change of coordinates we can assume without loss of generality that $x_{0}=0$ and $v=e_{n}$, and for simplicity we set $\mathbf{C}:=\mathbf{C}_{h^{\beta} / 2}\left(0, e_{n}\right)$. With this assumption we then have that for $s=t, t-h$

$$
\partial E_{s}^{(h)} \cap \mathbf{C}=\left\{\left(y, f_{s}(y)\right) \in \mathbb{R}^{n-1} \times \mathbb{R}:|y| \leq h^{\beta} / 2\right\}
$$


where $f_{s}: B_{h^{\beta} / 2} \subset \mathbb{R}^{n-1} \rightarrow \mathbb{R}$ are $C^{1, \kappa}$ functions with

$$
\left\|\nabla f_{S}\right\|_{L^{\infty}\left(B_{h^{\beta} / 2}\right)} \leq \omega(h)
$$

In view of Fubini's theorem it is then clear that

$$
\begin{gathered}
\int_{\mathbf{C}}\left(\chi_{E_{t}^{(h)}}-\chi_{E_{t-h}^{(h)}}\right) d x=\int_{B_{h^{\beta} / 2}}\left(f_{t}(y)-f_{t-h}(y)\right) d y, \\
\int_{\mathbf{C}}\left|\chi_{E_{t}^{(h)}}-\chi_{E_{t-h}^{(h)}}\right| d x=\int_{B_{h^{\beta} / 2}}\left|f_{t}(y)-f_{t-h}(y)\right| d y .
\end{gathered}
$$

Moreover, from (4.12) it follows that there exists a geometric constant $C>0$ such that, for every $y \in B_{h^{\beta} / 2}$,

$$
\left|\operatorname{sd}_{E_{t-h}^{(h)}}\left(y, f_{t}(y)\right) \sqrt{1+\left|\nabla f_{t}(y)\right|^{2}}-\left(f_{t}(y)-f_{t-h}(y)\right)\right| \leq C \omega(h)\left|f_{t}(y)-f_{t-h}(y)\right| .
$$

Therefore, one infers (4.11) as follows

$$
\begin{aligned}
& \left|\int_{\partial E_{t}^{(h)} \cap \mathbf{C}} \mathrm{sd}_{E_{t-h}^{(h)}} d \mathcal{H}^{n-1}-\int_{B_{h \beta} / 2}\left(f_{t}(y)-f_{t-h}(y)\right) d y\right| \\
& \quad=\left|\int_{B_{h \beta} / 2}\left(\operatorname{sd}_{E_{t-h}^{(h)}}\left(y, f_{t}(y)\right) \sqrt{1+\left|\nabla f_{t}(y)\right|^{2}}-\left(f_{t}(y)-f_{t-h}(y)\right)\right) d y\right| \\
& \quad \leq C \omega(h) \int_{B_{h \beta} / 2}\left|f_{t}-f_{t-h}\right| d y,
\end{aligned}
$$

where we used that (4.12).

We are finally ready for the proof of Proposition 4.1.

\subsection{Proof of Proposition 4.1}

We fix any time $t \in[2 h,+\infty)$. For every $x_{0} \in \partial E_{t}^{(h)}$, we fix $\alpha \in\left(\frac{1}{2}, \frac{n+2}{2(n+1)}\right)$ and consider the following open set $A_{x_{0}}$ defined as follows:

(i) if (4.6) holds, then we set $A_{x_{0}}:=\mathbf{C}_{h^{\beta} / 2}\left(x_{0}, v\right)$ where $v \in \mathbb{R}^{n}$ is the unit vector in Lemma 4.2;

(ii) otherwise we set $A_{x_{0}}:=B_{\gamma_{n} \sqrt{h}}\left(x_{0}\right)$.

Note that by Proposition 3.2 we have that $\left\{A_{x_{0}}\right\}_{x_{0} \in \partial E_{t}^{(h)}}$ is a covering of $E_{t}^{(h)} \Delta E_{t-h}^{(h)}$. Moreover, by a simple application of Besicovitch's covering theorem, cp. [16, Chapter 1.5.2] (applied, for example, to the balls to $B_{h^{\beta} / 2}\left(x_{0}\right) \subset A_{x_{0}}$ ), there exists a finite collections of points $I \subset \partial E_{t}^{(h)}$ such that $\left\{A_{x_{0}}\right\}_{x_{0} \in I}$ is a covering of $E_{t}^{(h)} \Delta E_{t-h}^{(h)}$.

We estimate the contribution of the integrals in (4.1) in every $A_{x_{0}}$ with $x_{0} \in I$ in two steps, depending on whether (i) above applies or (ii). 
Estimate in case (i). We use Corollary 4.3 and deduce that

$$
\begin{aligned}
& \left|\int_{A_{x_{0}}}\left(\chi_{E_{t}^{(h)}}-\chi_{E_{t-h}^{(h)}}\right) \phi d x-\int_{\partial E_{t}^{(h)} \cap A_{x_{0}}} \operatorname{sd}_{E_{t-h}^{(h)}} \phi d \mathcal{H}^{n-1}\right| \\
& \quad \leq\left|\phi\left(x_{0}\right)\right|\left|\int_{A_{x_{0}}}\left(\chi_{E_{t}^{(h)}}-\chi_{E_{t-h}^{(h)}}\right) d x-\int_{\partial E_{t}^{(h)} \cap A_{x_{0}}} \operatorname{sd}_{E_{t-h}^{(h)}} d \mathcal{H}^{n-1}\right| \\
& \quad+\left|\int_{A_{x_{0}}}\left(\chi_{E_{t}^{(h)}}-\chi_{E_{t-h}^{(h)}}\right)\left(\phi-\phi\left(x_{0}\right)\right) d x-\int_{\partial E_{t}^{(h)} \cap A_{x_{0}}} \operatorname{sd}_{E_{t-h}^{(h)}}\left(\phi-\phi\left(x_{0}\right)\right) d \mathcal{H}^{n-1}\right| \\
& \quad \leq C\left(\omega(h)\|\phi\|_{L^{\infty}}+h^{\beta}\|\nabla \phi\|_{L^{\infty}}\right) \int_{A_{x_{0}}}\left|\chi_{E_{t}^{(h)}}-\chi_{E_{t-h}^{(h)}}\right| d \mathcal{H}^{n-1},
\end{aligned}
$$

where we used the fact that $A_{x_{0}}=\mathbf{C}_{h^{\beta} / 2}\left(x_{0}, v\right)$.

Estimate in case (ii). By assumption there exists a point $y_{0} \in B_{\gamma_{n} \sqrt{h}}\left(x_{0}\right) \cap\left(E_{t}^{(h)} \Delta E_{t-h}^{(h)}\right)$ such that $\left|v^{(h)}\left(t, y_{0}\right)\right|>h^{\alpha-1}$. Without loss of generality we can assume that $y_{0} \in E_{t}^{(h)}$ (the other case can be treated analogously and we leave the details to the reader). It is then clear that $B_{h^{\alpha} / 2}\left(y_{0}\right) \subset \mathbb{R}^{n} \backslash E_{t-h}^{(h)}$ and $v^{(h)}(t, y)>h^{\alpha-1} / 2$ for every $y \in B_{h^{\alpha} / 2}\left(y_{0}\right)$. Since $h^{\alpha}<2 \gamma_{n} h^{1 / 2}$, we can apply the density estimate in (3.11) and deduce that

$$
C_{n} h^{(n+1) \alpha-1} \leq \int_{B_{h^{\alpha} / 2}\left(y_{0}\right) \cap\left(E_{t}^{(h)} \Delta E_{t-h}^{(h)}\right)}\left|v^{h}\right| d x .
$$

Similarly, by the density estimate in (3.12) and Proposition 3.2 we deduce that

$$
\int_{B_{\gamma_{n} \sqrt{h}}\left(x_{0}\right) \cap \partial E_{t}^{(h)}}\left|\mathrm{d}_{E_{t-h}^{h}}\right| d \mathcal{H}^{n-1} \leq C_{n} h^{n / 2} .
$$

From (4.14), (4.15) and $B_{h^{\alpha} / 2}\left(y_{0}\right) \subset B_{2 \gamma_{n} \sqrt{h}}\left(x_{0}\right)$ we then deduce that

$$
\begin{aligned}
\int_{A_{x_{0}}} \mid \chi_{E_{t}^{(h)}-} & \chi_{E_{t-h}^{(h)}} \mid+\int_{A_{x_{0}} \cap \partial E_{t}^{(h)}} \mathrm{d}_{E_{t-h}^{(h)}} d \mathcal{H}^{n-1} \\
& \leq C_{n} h^{n / 2-(n+1) \alpha+1} \int_{B_{2 \gamma_{n} \sqrt{h}}\left(x_{0}\right) \cap\left(E_{t}^{(h)} \Delta E_{t-h}^{(h)}\right)}\left|v^{h}\right| d x .
\end{aligned}
$$

We can then sum (4.13) and (4.16) over $x_{0} \in I$ and, recalling (3.15), (3.16) and (3.17), we get

$$
\begin{aligned}
& \left|\int\left(\chi_{E_{t}^{(h)}}-\chi_{E_{t-h}^{(h)}}\right) \phi d x-\int_{\partial E_{t}^{(h)}} \operatorname{sd}_{E_{t-h}^{(h)}} \phi d \mathcal{H}^{n-1}\right| \\
& \leq \sum_{x_{0} \in I}\left|\int_{A_{x_{0}}}\left(\chi_{E_{t}^{(h)}}-\chi_{E_{t-h}^{(h)}}\right) \phi d x-\int_{\partial E_{t}^{(h)} \cap A_{x_{0}}} \operatorname{sd}_{E_{t-h}^{(h)}} \phi d \mathcal{H}^{n-1}\right| \\
& \leq C_{n, 0}\left(\omega(h)\|\phi\|_{L^{\infty}}+h^{\beta}\|\nabla \phi\|_{L^{\infty}}+h^{n / 2-(n+1) \alpha+1}\|\phi\|_{L^{\infty}}\right) \\
& \quad \times\left(\left|E_{t}^{(h)} \Delta E_{t-h}^{(h)}\right|+\int_{E_{t}^{(h)} \Delta E_{t-h}^{(h)}}\left|v^{h}\right| d x\right)
\end{aligned}
$$

where we used the finite finiteness of the covering. 
Finally, integrating in time and using (3.18) and (3.19) we get

$$
\begin{aligned}
& \left|\int_{2 h}^{+\infty} \frac{1}{h}\left[\int_{E_{t}^{(h)}} \phi d x-\int_{E_{t-h}^{(h)}} \phi d x\right] d t-\int_{h}^{+\infty} \int_{\partial E_{t}^{(h)}} \phi v_{h} d \mathcal{H}^{n-1} d t\right| \\
& \leq C_{n, 0, T}\left(\omega(h)\|\phi\|_{L^{\infty}}+h^{\beta}\|\nabla \phi\|_{L^{\infty}}+h^{n / 2-(n+1) \alpha+1}\|\phi\|_{L^{\infty}}\right),
\end{aligned}
$$

where $T>0$ is such that $\operatorname{supp}(\phi) \subset[0, T] \times \mathbb{R}^{n}$. Recalling the definition of $\alpha$ and taking the limit as $h$ goes to 0 , we conclude (4.1).

Acknowledgments Open access funding provided by Max Planck Society (or associated institution if applicable). C.S. acknowledges the kind hospitality of the Max-Planck-Institut für Mathematik in den Naturwissenschaften in Leipzig, Germany, where part of this work was performed.

Open Access This article is distributed under the terms of the Creative Commons Attribution 4.0 International License (http://creativecommons.org/licenses/by/4.0/), which permits unrestricted use, distribution, and reproduction in any medium, provided you give appropriate credit to the original author(s) and the source, provide a link to the Creative Commons license, and indicate if changes were made.

\section{Appendix: A density lemma}

We premise the following density estimate for one-sided minimizers of the perimeter. The estimate can be easily deduced from the original arguments by De Giorgi exploited for minimizers [14].

Lemma 4.4 There exists a dimensional constant $c_{n}>0$ with this property. Let $E \subset \mathbb{R}^{n}$ be a set of finite perimeter, $R, \mu>0$ and $x_{0} \in \partial E$ be such that

$$
\operatorname{Per}(E) \leq \operatorname{Per}\left(E \cup B_{r}\left(x_{0}\right)\right)+\mu\left|B_{r}\left(x_{0}\right) \backslash E\right| \quad \forall 0<r<R .
$$

Then,

$$
c_{n} r^{n} \leq\left|B_{r}\left(x_{0}\right) \backslash E\right| \quad \forall 0<r<\min \left\{R, \mu^{-1}\right\} .
$$

Recall that $x_{0} \in \partial E$ if $\min \left\{\left|B_{r}\left(x_{0}\right) \backslash E\right|,\left|E \cap B_{r}\left(x_{0}\right)\right|\right\}>0$ for every $r>0$.

Proof Without loss of generality, we can assume $x_{0}=0$. We use the following indentity which are true a.e. $r>0$ :

$$
\begin{aligned}
\operatorname{Per}\left(E \cup B_{r}\right) & =\mathcal{H}^{n-1}\left(\partial B_{r} \backslash E\right)+\operatorname{Per}\left(E, \mathbb{R}^{n} \backslash B_{r}(x)\right), \\
\operatorname{Per}\left(B_{r} \backslash E\right) & =\mathcal{H}^{n-1}\left(\partial B_{r} \backslash E\right)+\operatorname{Per}\left(E, B_{r}\right), \\
\operatorname{Per}(E) & =\operatorname{Per}\left(E, B_{r}\right)+\operatorname{Per}\left(E, \mathbb{R}^{n} \backslash B_{r}\right) .
\end{aligned}
$$

Indeed, if $E$ were smooth, these formulas follow for all the $r$ such that $B_{r}$ and $E$ have transversal intersections. Otherwise one can argue by approximation. Using now (4.18), we deduce that, for a.e. $r>0$,

$$
\begin{aligned}
& \operatorname{Per}\left(B_{r} \backslash E\right) \stackrel{(A .4)}{=} \mathcal{H}^{n-1}\left(\partial B_{r} \backslash E\right)+\operatorname{Per}\left(E, B_{r}\right) \\
& \stackrel{(A .5)}{\leq} \mathcal{H}^{n-1}\left(\partial B_{r} \backslash E\right)+\operatorname{Per}(E)-\operatorname{Per}\left(E, \mathbb{R}^{n} \backslash B_{r}\right) \\
& \text { (A.1) } \underset{\leq}{\&}(A .3) 2 \mathcal{H}^{n-1}\left(\partial B_{r} \backslash E\right)+\mu\left|B_{r} \backslash E\right| \text {. }
\end{aligned}
$$


By the isoperimetric inequality [19, Corollary 1.29], there exists a dimensional constant $C>0$, such that

$$
C\left|B_{r} \backslash E\right|^{\frac{n-1}{n}} \leq \operatorname{Per}\left(B_{r} \backslash E\right) \stackrel{(A .6)}{\leq} 2 \mathcal{H}^{n-1}\left(\partial B_{r} \backslash E\right)+\mu\left|B_{r} \backslash E\right| .
$$

Setting $f(r):=\left|B_{r} \backslash E\right|$, by the coarea formula [16, 3.4.4], it holds

$$
\mathcal{H}^{n-1}\left(\partial B_{r} \backslash E\right)=f^{\prime}(r) \text { for a.e. } r>0 .
$$

Hence, (4.24) reads as

$$
C f(r)^{\frac{n-1}{n}} \leq 2 f^{\prime}(r)+\mu f(r) .
$$

Finally, note that $f(r) \leq \omega_{n} r^{n}$, from which $f(r) \leq \omega_{n}^{1 / n} r f(r)^{n-1 / n}$. Therefore, there exists a dimensional constant $C_{n}>0$ such that if $0<r<\min \left\{R, C_{n} \mu^{-1}\right\}$, then the last term in (4.25) can be absorbed in the left hand side and deduce that

$$
f(r)^{\frac{n-1}{n}} \leq C f^{\prime}(r) .
$$

Integrating (4.24) (recall that $f(r)>0$ for every $r>0$ ) we get the desired (4.19) for every $0<r<\min \left\{R, C_{n} \mu^{-1}\right\}$ and, by changing the dimensional constant $c_{n}>0$, for every $0<r<\min \left\{R, \mu^{-1}\right\}$.

\section{References}

1. Alfaro, M., Alifrangis, P.: Convergence of a mass conserving Allen-Cahn equation whose lagrange multiplier is nonlocal and local. Interfaces Free Bound 16, 243-268 (2014)

2. Almgren, F., Taylor, J.E., Wang, L.: Curvature-driven flows: a variational approach. SIAM J. Control Optim. 31(2), 387-438 (1993)

3. Ambrosio, L., Fusco, N., Pallara, D.: Functions of Bounded Variation and Free Discontinuity Problems. Oxford Mathematical Monographs. The Clarendon Press, Oxford University Press, New York (2000)

4. Andrews, B.: Volume-preserving anisotropic mean curvature flow. Indiana Univ. Math. J. 50(2), 783-827 (2001)

5. Bellettini, G., Caselles, V., Chambolle, A., Novaga, M.: The volume preserving crystalline mean curvature flow of convex sets in $\mathbb{R}^{N}$. J. Math. Pure Appl. 92(5), 499-527 (2009)

6. Bronsard, L., Stoth, B.: Volume-preserving mean curvature flow as a limit of a nonlocal Ginzburg-Landau equation. SIAM J. Math. Anal. 28(4), 769-807 (1997)

7. Cabezas-Rivas, E., Miquel, V.: Volume preserving mean curvature flow in the hyperbolic space. Indiana Univ. Math. J. 56(5), 2061-2086 (2007)

8. Cabezas-Rivas, E., Miquel, V.: Volume preserving mean curvature flow of revolution hypersurfaces between two equidistants. Calc. Var. Partial Differ. Equ. 43(1-2), 185-210 (2012)

9. Caginalp, G.: Stefan and hele-shaw type models as asymptotic limits of the phase-field equations. Phys. Rev. A 39(11), 5887-5896 (1989)

10. Capuzzo Dolcetta, I., Finzi Vita, S., March, R.: Area-preserving curve-shortening flows: from phase separation to image processing. Interface Free Bound 4(4), 325-343 (2002)

11. Carter, W., Roosen, A., Cahn, J., Taylor, J.: Shape evolution by surface diffusion and surface attachment limited kinetics on completely faceted surfaces. Acta Metall. Mater. 43(12), 4309-4323 (1995)

12. Chen, X., Hilhorst, D., Logak, E.: Mass conserving Allen-Cahn equation and volume preserving mean curvature flow. Interface Free Bound 12(4), 527-549 (2010)

13. Dai, S., Niethammer, B., Pego, R.L.: Crossover in coarsening rates for the monopole approximation of the Mullins-Sekerka model with kinetic drag. In: Proceedings of the Royal Society of Edinburgh: Section A Mathematics vol. 140(03), pp. 553-571 (2010)

14. De Giorgi, E.: Nuovi teoremi relativi alle misure $(r-1)$-dimensionali in uno spazio ad $r$ dimensioni. Ricerche Mat 4, 95-113 (1955)

15. Escher, J., Simonett, G.: The volume preserving mean curvature flow near spheres. Proc. Am. Math. Soc. 126(9), 2789-2796 (1998)

16. Evans, L.C., Gariepy, R.F.: Measure Theory and Fine Properties of Functions. Studies in Advanced Mathematics. CRC Press, Boca Raton, FL (1992) 
17. Gage, M.: On an area-preserving evolution equation for plane curves. In: Iannelli, M., Nagel, R., Piazzera, S. (eds), Nonlinear Problems in Geometry (Mobile, Ala., 1985), volume 51 of Contemp. Math. pp 52-62. Am. Math. Soc. (1986)

18. Gilbarg, D., Trudinger, N.S.: Elliptic Partial Differential Equations of Second Order. Classics in Mathematics (Reprint of the 1998 edition). Springer, Berlin (2001)

19. Giusti, E.: Minimal Surfaces and Functions of Bounded Variation. Monographs in Mathematics, 80th edn. Birkhäuser Verlag, Basel (1984)

20. Goldman, M., Novaga, M.: Volume-constrained minimizers for the prescribed curvature problem in periodic media. Calc. Var. Partial Differ. Equ. 44(3-4), 297-318 (2012)

21. Gurtin, M.E.: Thermomechanics of Evolving Phase Boundaries in the Plane. Oxford Mathematical Monographs. The Clarendon Press, Oxford University Press, New York (1993)

22. Huisken, G.: The volume preserving mean curvature flow. J. Rein. Angew. Math. 382, 35-48 (1987)

23. Hutchinson, J.E.: Second fundamental form for varifolds and the existence of surfaces minimising curvature. Indiana Univ. Math. J. 35(1), 45-71 (1986)

24. Li, H.: The volume-preserving mean curvature flow in Euclidean space. Pac. J. Math. 243(2), 331-355 (2009)

25. Lifshitz, I.M., Slyozov, V.V.: The kinetics of precipitation from supersaturated solid solutions. J. Phys. Chem. Solids 19(1-2), 35-50 (1961)

26. Luckhaus, S., Sturzenhecker, T.: Implicit time discretization for the mean curvature flow equation. Calc. Var. Partial Differ. Equ. 3(2), 253-271 (1995)

27. Maggi, F.: Sets of finite perimeter and geometric variational problems, volume 135 of Cambridge Studies in Advanced Mathematics. An introduction to geometric measure theory. Cambridge University Press, Cambridge (2012)

28. Michor, P.W., Mumford, D.: Riemannian geometries on spaces of plane curves. J. Eur. Math. Soc. (JEMS) 8(1), 1-48 (2006)

29. Mugnai, L., Seis, C.: On the coarsening rates for attachment-limited kinetics. SIAM J. Math. Anal. 45(1), 324-344 (2013)

30. Peng, D., Merriman, B., Osher, S., Zhao, H., Kang, M.: A pde-based fast local level set method. J. Comput. Phys. 155(2), 410-438 (1999)

31. Ruuth, S.J., Wetton, B.T.R.: A simple scheme for volume-preserving motion by mean curvature. J. Sci. Comput. 19(1-3), 373-384 (2003). Special issue in honor of the sixtieth birthday of Stanley Osher

32. Tarshis, L.A., Walker, J.L., Gigliotti, M.F.X.: Solidification. Annu. Rev. Mater. Sci. 2(1), 181-216 (1972)

33. Wagner, C.: Theorie der Alterung von Niederschlägen durch Umlösen (Ostwald-Reifung). Zeitschrift für Elektrochemie, Berichte der Bunsengesellschaft für physikalische Chemie 65(7-8), 581-591 (1961) 